\title{
Time-dependent search of neutrino emission from $X$-ray binaries with the ANTARES telescopes
}

\section{Damien Dornic*}

Aix Marseille Université, CNRS/IN2P3, CPPM UMR 7346, 13288, Marseille, France,

E-mail: dornic@eppp.in2p3.fr

\section{A. Sánchez-Losa}

IFIC - Instituto de Física Corpuscular, Edificios Investigaciòn de Paterna, CSIC - Universitat de Valencia, Apdo. de Correos 22085, 46071 Valencia, Spain

E-mail: agustin.sanchez@ific.uv.es

\section{on behalf the ANTARES Collaboration}

\begin{abstract}
ANTARES is currently the largest neutrino telescope operating in the Northern Hemisphere, aiming at the detection of high-energy neutrinos from astrophysical sources. By design, neutrino telescopes constantly monitor at least one complete hemisphere of the sky and are thus well set to detect neutrinos produced in transient astrophysical sources. The flux of high-energy neutrinos from transient sources is expected to be lower than the one expected from steady sources, but the background originating from interactions of charged cosmic rays in the Earth's atmosphere can be drastically reduced by requiring a directional and temporal coincidence of the astrophysical phenomenon detected by a satellite. The time-dependent point-source search has been applied to a list of 34 x-ray binary systems while observed in high flaring activities in the 2008-2012 satellite data, RXTE/ASM, MAXI and Swift/BAT. The results of this search are presented together with the comparison between the neutrino flux upper-limits with the measured gamma-ray spectral energy distribution and the prediction from astrophysical models.
\end{abstract}

The 34th International Cosmic Ray Conference,

30 July- 6 August, 2015

The Hague, The Netherlands

\footnotetext{
* Speaker.
} 


\section{Introduction}

Neutrinos are unique messengers for studying the high-energy Universe as they are neutral, stable, interact weakly, and travel directly from their sources without absorption or deflection. Therefore, the reconstruction of the arrival directions of cosmic neutrinos would allow both the sources of the cosmic rays - supernova remnant shocks, active galactic nuclei jets, x-ray binary jets, gamma-ray bursts, etc. [1] - and the relevant acceleration mechanisms acting within them to be identified.

$\mathrm{X}$-ray binaries (XRB) are a class of binary stars that are luminous in X-rays. The x-rays are produced by matter falling from the donor (usually a relatively normal star), to the accretor, which is compact: a white dwarf, neutron star (NS), or black hole (BH). These systems are usually classified as low-mass x-ray binary (LMXB) and high-mass x-ray binary (HMXB), depending on the mass of the donor. In very few cases, the presence of relativistic jets has been confirmed by radio measurements. The jet signature may be present in all the XRB sources. However, the composition of the jets is still unknown. Their spectral energy distribution can be described by two components: a low-energy one from radio to X-rays and a high-energy one from X-rays to very high-energy gamma rays. The non-thermal emission is probably dominated by leptonic processes of accelerated eletrons but a hadronic component could also be present. In hadronic models, associated with the very high-energy gamma rays from $\pi^{0}$ decays, the decay of the charged pions gives rise to a correlated neutrino emission. Up to now, in only three cases, a hadronic component has been identified by spectroscopy (detection of iron or nikel lines) [2, 3]. Several authors have estimated the flux of high-energy neutrino coming from XRB, resulting in very different shapes and normalisations $[4,5,6]$. To cover the majority of the range allowed by the models accessible to the ANTARES sensitivity, three neutrino-energy spectra are tested in this analysis: $E^{-2}$, $E^{-2} \exp (-E / 100 \mathrm{TeV})$ and $E^{-2} \exp (-E / 10 \mathrm{TeV})$, where $E$ is the neutrino energy.

In the ANTARES telescope [7], events are primarily detected underwater by observing the Cherenkov light induced by relativistic muons in the darkness of the deep sea. Owing to their low interaction probablility, only neutrinos have the ability to cross the Earth. Therefore, an upgoing muon is an unambiguous signature of a neutrino interaction close to the detector. To distinguish astrophysical neutrino events from background events (muons and neutrinos) generated in the atmosphere, energy and direction reconstructions have been used in several searches [8] [9]. To improve the signal-to-noise discrimination, the arrival time information can be used, significantly reducing the effective background [10].

In this paper, the results of a time-dependent search for cosmic neutrino sources using the ANTARES data taken from 2008 to 2012 is presented. This extends a previous ANTARES analysis [11] where only five sources and the first three years were considered. The analysis is applied to a list of promising X-ray binaries candidates detected by various satellites such as Swift, RXTE, MAXI and Fermi. Section 2 and 3 present the algorithms to identify the outburst periods and the statistical method adopted for this analysis, respectively. Section 4 summarised the results of this search. Conclusions are drawn in Section 5. 


\section{Selection of outburst periods}

The time-dependent analysis described in the following section is applied to a list of x-ray binaries exhibiting outburst periods in their light-curves. The light curves are obtained mainly using the Swift/BAT telescope ${ }^{1}$. These data are complemented by the data from others instruments: RXTE/ASM ${ }^{2}, \mathrm{MAXI}^{3}$ and Fermi/GBM ${ }^{4}$. A maximum likelihood block (MLB) algorithm [12] is used to remove noise from the light curve by iterating over the data points and selecting periods during which data are consistent with a constant flux within statistical errors. This algorithm is applied independently to all the light curves from all the satellites. Depending on the time period and the avaliability of the different instruments, the outbursts are more defined in one sample compare to the others. As the energy range and the sensitivity of these telescopes are different, it is not easy to merge the flares of each sources. The value of the steady state (i.e. baseline, BL) and its fluctuation $\left(\sigma_{B L}\right)$ are determined with a Gaussian fit of the lower part of the distribution of the flux data points. The baseline is removed from the light curve and the amplitude is converted to a relative amplitude using the sigma of the baseline fluctuations. Finally, the relative light curves from different instruments are merged.

The flaring periods are defined in three main steps. Firstly, seeds are identified by searching for points with an amplitude, or blocks with a fluence above $B L+8 \sigma_{B L}$. Then, each period is extended forward and backward up to an emission compatible with $B L+1 \sigma_{B L}$. An additional delay of 0.5 days is added before and after the flare in order to take into account that the precise time of the flare is not known (one-day binned light curve). Finally, spurious flares are discarded if they are not visible by at least one other intrument. The final list includes $34 \mathrm{x}$-ray binaries: 1 HMXB (BH), 12 HMXB (NS), 8 HMXB (BH candidate), 10 LMXB (NS), 3 XRB (BH candidate). The main characteristics of these XRB are reported in Table 1.

\section{Time-dependent analysis}

The ANTARES data collected between 2008 and 2012, corresponding to 1044 days of livetime, are analysed to search for neutrino events around the selected sources, in coincidence with the time periods defined in the previous section. The statistical method adopted to infer the presence of a signal on top of the atmospheric neutrino background, or alternatively set upper limits on the neutrino flux is an unbinned method based on a likelihood ratio test statistic. The likelihood, $\mathscr{L}$, is defined as:

$$
\ln \mathscr{L}=\left(\sum_{i=1}^{N} \ln \left[\mathscr{N}_{\mathrm{S}} \mathscr{S}_{i}+\mathscr{N}_{\mathrm{B}} \mathscr{B}_{i}\right]\right)-\left[\mathscr{N}_{\mathrm{S}}+\mathscr{N}_{\mathrm{B}}\right]
$$

where $\mathscr{S}_{i}$ and $\mathscr{B}_{i}$ are the probabilities for signal and background for an event i, respectively, $\mathscr{N}_{\mathrm{s}}$ (unknown) and $\mathscr{N}_{\mathrm{B}}$ (known) are the number of expected signal and background event in the data sample. To discriminate the signal-like events from the background ones, these probabilities are

\footnotetext{
${ }^{1}$ http : //swift.gsfc.nasa.gov/results/transients

${ }^{2}$ http : //xte.mit.edi/ASMlc.html

${ }^{3}$ http : //maxi.riken.jp

${ }^{4}$ http: //heasarc.gsfc.nasa.gov/W3Browse/fermi/fermigdays.html
} 
Table 1: List of $34 \mathrm{X}$-ray binaries with significant flares selected for this analysis.

\begin{tabular}{|c||c|c|c|}
\hline Name & Class & RA [ $\left.{ }^{\circ}\right]$ & Dec $\left[^{\circ}\right]$ \\
\hline \hline Cyg X-1 & HMXB (BH) & 230.170 & -57.167 \\
\hline 1A0535p262 & HMXB (NS) & 84.727 & 26.316 \\
\hline 1A1118-61 & HMXB (NS) & 170.238 & -61.917 \\
\hline Ginga 1843p00 & HMXB (NS) & 281.404 & 0.863 \\
\hline GS 0834-430 & HMXB (NS) & 128.979 & -43.185 \\
\hline GX 304-1 & HMXB (NS) & 195.321 & -61.602 \\
\hline H 1417-624 & HMXB (NS) & 215.300 & -62.70 \\
\hline MXB 0656-072 & HMXB (NS) & 104.572 & -7.210 \\
\hline XTE J1946p274 & HMXB (NS) & 296.414 & 27.365 \\
\hline Cyg X-3 & HMXB (NS) & 308.107 & 40.958 \\
\hline GX 1p4 & HMXB (NS) & 263.009 & -24.746 \\
\hline MAXI J1409-619 & HMXB (NS) & 212.011 & -61.984 \\
\hline GRO J1008-57 & HMXB (NS) & 152.433 & -58.295 \\
\hline GX 339-4 & LMXB (BHC) & 255.7 & -48.8 \\
\hline 4U 1630-472 & LMXB (BHC) & 248.504 & -47.393 \\
\hline IGR J17091-3624 & LMXB (BHC) & 257.282 & -36.407 \\
\hline IGR J17464-3213 & LMXB (BHC) & 266.565 & -32.234 \\
\hline MAXI J1659-152 & LMXB (BHC) & 254.757 & -15.258 \\
\hline SWIFT J1910.2-0546 & LMXB (BHC) & 287.595 & -5.799 \\
\hline XTE J1752-223 & LMXB (BHC) & 268.063 & -22.342 \\
\hline SWIFT J1539.2-6227 & LMXB (BHC) & 234.800 & -62.467 \\
\hline 4U 1954p31 & LMXB (NS) & 298.926 & 32.097 \\
\hline Aq1 X-1 & LMXB (NS) & 287.817 & 0.585 \\
\hline Cir X-1 & LMXB (NS) & 230.170 & -57.167 \\
\hline EXO 1745-248 & LMXB (NS) & 267.022 & -24.780 \\
\hline H 1608-522 & LMXB (NS) & 243.179 & -52.423 \\
\hline SAX J1808.4-3658 & LMXB (NS) & 272.115 & -36.977 \\
\hline XTE J1810-189 & LMXB (NS) & 272.586 & -19.070 \\
\hline 4U 1636-536 & LMXB (NS) & 250.231 & -53.751 \\
\hline 4U 1705-440 & LMXB (NS) & 257.225 & -44.102 \\
\hline IGR J17473-2721 & LMXB (NS) & 266.825 & -27.344 \\
\hline MAXI J1836-194 & XRB (BHC) & 278.931 & -19.320 \\
\hline XTE J1652-453 & XRB (BHC) & 253.085 & -45.344 \\
\hline SWIFT J1842.5-1124 & XRB (BHC) & $280.573 ~$ & -11.418 \\
\hline
\end{tabular}

described by the product of three components related to the direction, energy, and timing of each event. For an event $i$, the signal probability is:

$$
\mathscr{S}_{i}=\mathscr{S}^{\text {space }}\left(\Psi_{i}\left(\alpha_{s}, \delta_{s}\right)\right) \cdot \mathscr{S}^{\text {energy }}\left(d E / d X_{i}\right) \cdot \mathscr{S}^{\text {time }}\left(t_{i}+l a g\right)
$$


where $\mathscr{S}^{\text {space }}$ is a parameterisation of the point spread function, i.e., $\mathscr{S}^{\text {space }}\left(\Psi_{i}\left(\alpha_{s}, \delta_{s}\right)\right)$ the probability to reconstruct an event $i$ at an angular distance $\Psi_{i}$ from the true source location $\left(\alpha_{s}, \delta_{s}\right)$. The energy PDF $\mathscr{S}^{\text {energy }}$ is parametrised with the normalised distribution of the muon energy estimator, $\mathrm{dE} / \mathrm{dX}$, of an event according to the studied energy spectrum. The shape of the time PDF, $\mathscr{S}^{\text {time }}$, for the signal event is extracted directly from the gamma-ray light curve assuming the proportionality between the gamma-ray and the neutrino fluxes. A possible lag of up to \pm 5 days has been introduced in the likelihood to allow for small lags in the proportionality. This corresponds to a possible shift of the entire time PDF. The lag parameter is fitted in the likelihood maximisation together with the number of fitted signal events in the data. The background probability for an event $i$ is:

$$
\mathscr{B}_{i}=\mathscr{B}^{\text {space }}\left(\delta_{i}\right) \cdot \mathscr{B}^{\text {energy }}\left(d E / d X_{i}\right) \cdot \mathscr{B}^{\text {time }}\left(t_{i}\right)
$$

where the directional PDF $\mathscr{B}^{\text {space }}$, the energy PDF $\mathscr{B}^{\text {energy }}$ and the time PDF $\mathscr{B}^{\text {time }}$ for the background are derived from data using, respectively, the observed declination distribution of selected events in the sample, the measured distribution of the energy estimator, and the observed time distribution of all the reconstructed muons.

The goal of the unbinned search is to determine, in a given direction in the sky and at a given time, the relative contribution of each component, and to calculate the probability to have a signal above a given background model. This is done via the test statistic, $\lambda$, defined as the ratio of the probability for the hypothesis of background and signal $\left(H_{\text {sig }+ \text { bkg }}\right)$ over the probability of only background $\left(H_{\mathrm{bkg}}\right)$ :

$$
\lambda=\sum_{i=1}^{N} \ln \frac{\mathscr{P}\left(x_{i} \mid H_{\mathrm{sig}+\mathrm{bkg}}\left(\mathscr{N}_{\mathrm{S}}\right)\right)}{\mathscr{P}\left(x_{i} \mid H_{\mathrm{bkg}}\right)}
$$

where $N$ is the total number of events in the considered data sample and $x_{i}$ are the observed event properties $\left(\delta_{i}, R A_{i}, d E / d X_{i}\right.$ and $\left.t_{i}\right)$. The evaluation of the test statistic is performed by generating pseudo-experiments simulating background and signal in a $30^{\circ}$ cone around the considered source according to the background-only and background plus signal hypotheses. The performance of the time-dependent analysis is computed with a toy experiment with a source assuming a squareshaped flare with a varying width assuming a flat background period. For time ranges characteristic of flaring activity, the time-dependent search presented here improves the discovery potential by on-average a factor 2-3 with respect to a standard time-integrated point-source search [8] under the assumption that the neutrino emission is correlated with the gamma-ray flaring activity.

\section{Results}

The results of the search is summarised in Table 2. Only two sources, GX1+4 and IGRJ170913624 , have a pre-trial p-value lower than $10 \%$. The lowest p-value, $4.1 \%$, is obtained for GX1+4 where one (three) event is coincident in a cone of 1(3) degres with large outbursts detected by Fermi/LAT. Figure 1 shows the light curve of GX $1+4$ with the time of the neutrino events, the estimated energy distribution, and the angular distribution of the events around the position of this source. The post-trial probability, computed by taking into account the 34 searches, is $72 \%$, and is thus compatible with background fluctuations. 
Table 2: Results of the search for neutrinos in coincidence with XRB outbursts. The total duration of all identified flares $\Delta t$, the optimised $\Lambda_{o p t}$ cuts, the number of required events for a $3 \sigma$ discovery $\left(N_{3 \sigma}\right)$ pre-trial, the number of fitted signal events by the likelihood $\left(N_{f i t}\right)$, the fitted time lag $(\mathrm{Lag})$ and the corresponding pre-trial (post-trial) probability are given together with the energy spectra.

\begin{tabular}{|c|c|c|c|c|c|c|c|c|}
\hline Source & $\Delta t$ & $\Lambda_{\text {opt }}$ & $N_{3 \sigma}$ & $N_{\text {fit }}$ & Lag & P-value & Post-trial & Spectrum \\
\hline \hline GX1+4 & $660 \mathrm{~d}$ & -5.2 & 2.45 & 0.69 & $-5 \mathrm{~d}$ & 0.041 & 0.72 & cutoff $100 \mathrm{TeV}$ \\
\hline IGRJ17091-3624 & $62 \mathrm{~d}$ & -5.4 & 1.75 & 0.31 & $+4 \mathrm{~d}$ & 0.065 & 0.94 & cutoff $10 \mathrm{TeV}$ \\
\hline
\end{tabular}
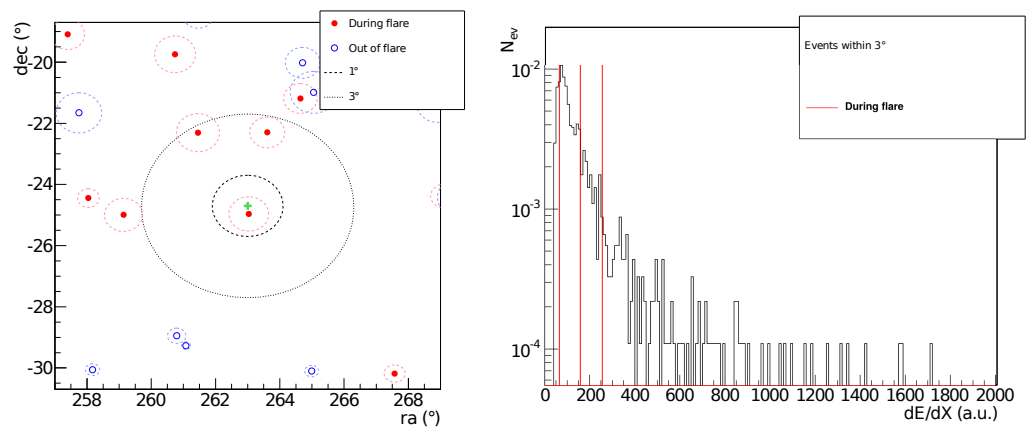

PRELIMINARY

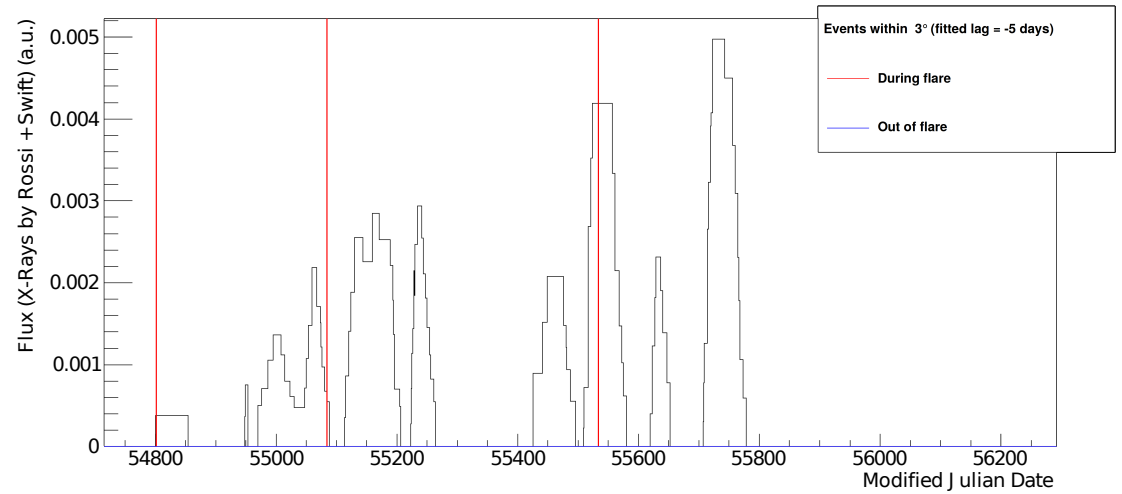

Figure 1: Results for GX 1+4. (Top left) Event map around the direction of GX1+4 indicated by the green cross. The full red (hollow blue) dots indicate the events (not) in time coincidence with the selected flares. The size of the circle around the dots is proportional to the estimated angular uncertainty for each event. (Top right) Distribution of the energy estimator $\mathrm{dE} / \mathrm{dX}$ in a $\pm 10^{\circ}$ declination band around the source direction. The red line displays the value of the event in coincidence with the flare in a $1^{\circ}$ cone around the source direction. (Bottom) Time PDF for the signal simulation (proportional to the x-ray light curve). The red line displays the times of the ANTARES events associated with the source during a flaring state in a $3^{\circ}$ box around the source position.

In the absence of a discovery, upper limits on the neutrino fluence, $\mathscr{F}_{v}$, at $90 \%$ confidence level are computed using 5-95\% of the energy range and the total effective flare duration. The limits are calculated according to the classical (frequentist) method for upper limits [13]. Figure 2 displays these upper limits. Systematic uncertainties of $15 \%$ on the angular resolution and $15 \%$ on the detector acceptance have beenincluded in the upper limit calculations.

The neutrino flux prediction for five microquasars have been computed according to the model [4] 

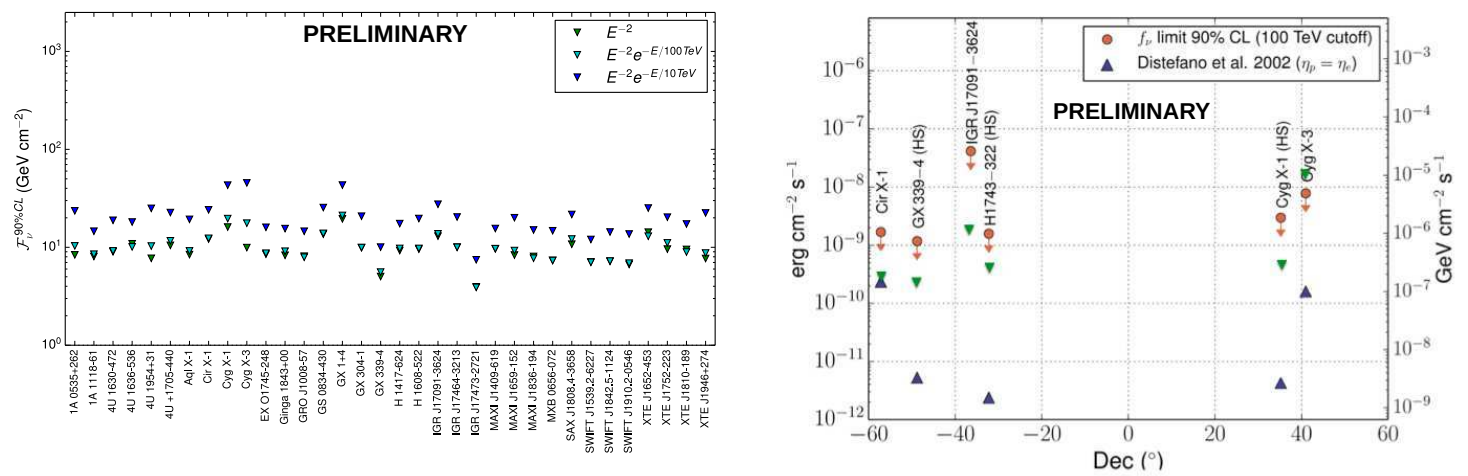

Figure 2: Left: upper limits on the neutrino fluence for the 34 studied XRB in the case of $E^{-2}$ (green triangle), $E^{-2} \exp (-E / 100 \mathrm{TeV})$ (cyan triangle), $E^{-2} \exp (-E / 10 \mathrm{TeV})$ (blue triangle) neutrino energy spectra. Right: upper limits at $90 \%$ C.L. on the energy flux in neutrinos obtained in this analysis considering a flux $\left.E^{-2} \exp (-\sqrt{(} E / 100 \mathrm{TeV})\right)($ circles), compared with the expectations by Ref. [4] in the case equipartition between electrons and protons (triangles).

using the latest measurements of the distance and of the jet parameters of the microquasars. Figure 3 (left) displays these predictions togethers with the upper limits computed for this analysis. For Cir X-1, the prediction is less than a factor 2 bellow the ANTARES upper limit. In Ref. [14], the authors have provided a calculation of the high-energy neutrino emission from GX339-4 in the hypothesis that the primary spectrum of the injected particles in the jets has spectral indexes = $-1.8 ;-2.0$ and that the ratio between proton and electron energy is equal to 1 and 100 , respectively (Figure 3 (middle)). The model with a ratio equal to 100 is excluded by the present limit. Finally, Figure 3 (right) shows the comparison between the neutrino flux expectations from Cyg X-3 provided by [15] and [16] and the computed upper limits. The upper limit does not allow to constrain these types of models.

\section{Conclusion}

This paper discusses the time-dependent search for cosmic neutrinos from x-ray binaries using the data taken with the full ANTARES detector between 2008 and 2012. These searches have been applied to a list of 34 XRB sources. The searches did not result in a statistically significant excess above the expected background from atmospheric neutrino and muon events. The most significant correlation was found for the source GX 1+4 for which few neutrino events was detected in time/spatial coincidence with the x-ray emission. However, the post-trial probability is of $72 \%$, thus compatible with the background fluctuations. The comparison with predictions from several models have shown that for some sources, the upper limits are closed from the expectations. Therefore, with additional data from ANTARES and with the order of magnitude sensitivity improvement expected from the next generation neutrino telescope, $\mathrm{KM}_{3 \mathrm{NeT}}{ }^{5}$, the prospects for future searches for neutrino emission from $\mathrm{x}$-ray binaries are very promising.

\footnotetext{
${ }^{5}$ http : //www.km3net.org
} 

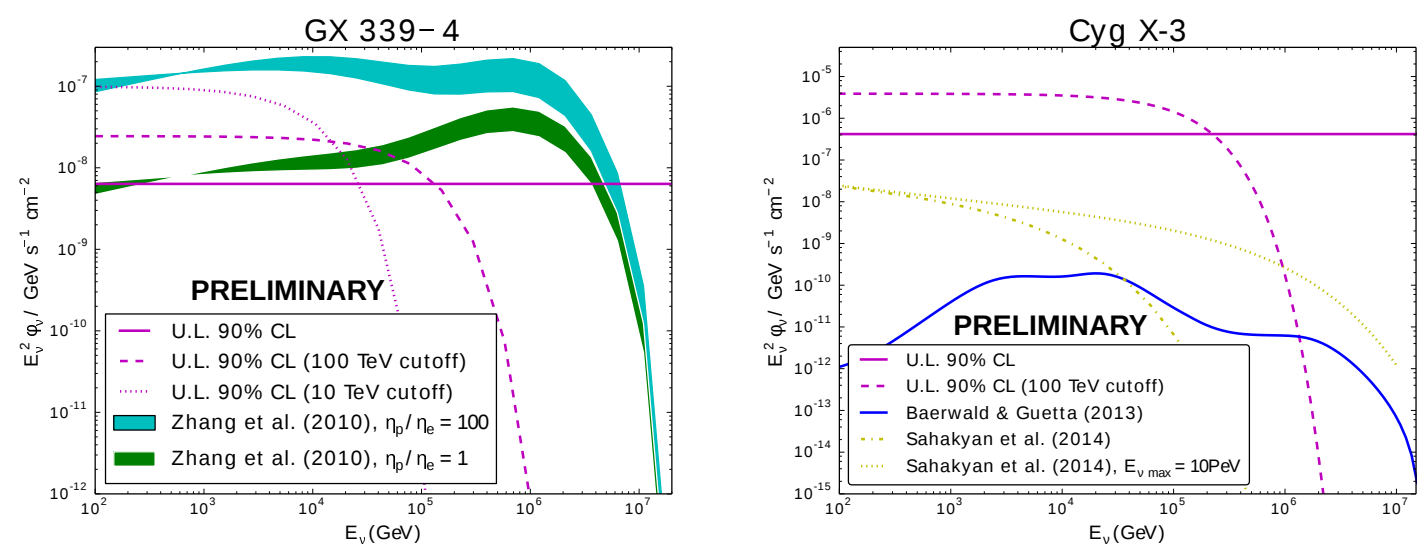

Figure 3: Left: upper limits on the neutrino flux for GX 339-4 in both the hypotheses without and with the cutoff at $100 \mathrm{TeV}$, compared to the prediction by the authors of Ref. [14] for a spectral index of the injected particles $-1.8<\alpha<-2.0$ and the ratio $n_{p}=n_{e}$ equal to 1 and 100 , respectively. Right: Upper limits on the neutrino flux for Cyg X-3 in both the hypotheses without and with the cutoff at $100 \mathrm{TeV}$, compared to the predictions by Ref.[15] and [16].

\section{References}

[1] J.K. Becker 2008, Phys. Rep., 458, 173.

[2] S. Migliari, R. Fender, M. Mendez, 2002, Science, 297, 1673.

[3] M.D. Trigo, J.C.A. Miller-Jones, S. Migliari, J.W. Broderick, T. Tzioumis, 2013, Nature, 504, 260.

[4] C. Distefano, D. Guetta, E. Waxman, A. Levinson, 2002, ApJ, 575, 378.

[5] G.E. Romero, D.F. Torres, M.M. Kaufman Bernadò, I.F. Mirabel, 2003, A\&A, 410, L1.

[6] A. Levinson, E. Waxman, Physical Review Letters, 2001, 87, 171101.

[7] M. Ageron et al., Nucl. Instrum. Meth. A 656 (2011) 11-38.

[8] S. Adrián-Martínez et al., The Astrophysical Journal 760:53(2012).

[9] J.A. Aguilar et al., Phys. Lett. B 696 (2011) 16-22.

[10] S. Adrián-Martínez et al., A\&A 559, A9 (2013)

[11] S. Adrián-Martínez et al. Journal of High Energy Astrophysics 3-4(2014) 9-17.

[12] J.D. Scargle, The Astrophysical Journal Supplement Series, 45, 1-71, 1981; J.D. Scargle, Astrophys. J., 504, 1998, 405-418; J.D. Scargle et al., Astrophys.J. 764 (2013) 167.

[13] J. Neyman, 1937, Phil. Trans. Royal Soc. London, Series A, 236, 333.

[14] J.F. Zhang, Y.G. Feng, M.C. Lei, Y.Y. Tang, Y.P. Tian, 2010, MNRAS, 407, 2468,

[15] P. Baerwald, D. Guetta, 2013, ApJ, 773,159.

[16] N. Sahakyan, G. Piano, M. Tavani, 2014, ApJ, 745, L7. 